CTP TAMU-51/96

$\mathrm{IC} / 96 / 211$

hep-th/9610107

\title{
Cosmological Solutions in String Theories
}

\author{
H. Lü ${ }^{\dagger}$, S. Mukherji ${ }^{\ddagger}$, C.N. Pope ${ }^{\dagger}$ \\ Center for Theoretical Physics, Texas A\&M University, College Station, Texas 77843 \\ K.-W. Xu \\ International Center for Theoretical Physics, Trieste, Italy \\ and \\ Institute of Modern Physics, Nanchang University, Nanchang, China
}

\begin{abstract}
$\underline{\text { ABSTRACT }}$
We obtain a large class of cosmological solutions in the toroidally-compactified low energy limits of string theories in $D$ dimensions. We consider solutions where a $p$-dimensional subset of the spatial coordinates, parameterising a flat space, a sphere, or an hyperboloid, describes the spatial sections of the physically-observed universe. The equations of motion reduce to Liouville or $S L(N+1, R)$ Toda equations, which are exactly solvable. We study some of the cases in detail, and find that under suitable conditions they can describe four-dimensional expanding universes. We discuss also how the solutions in $D$ dimensions behave upon oxidation back to the $D=10$ string theory or $D=11$ M-theory.
\end{abstract}

\footnotetext{
$\dagger$ Research supported in part by DOE grant DE-FG05-91-ER40633

$\ddagger$ Research supported in part by NSF grant PHY-9411543
} 


\section{Introduction}

The study of cosmological consequences of string theory has been an area of much active research in the past [1-5]. Recently, exploiting certain duality symmetry, it has been suggested that inflation can occur in string theory without relying on the potential energy density of the dilaton field [6, 7]. Since then, several papers have investigated aspects of string-inspired cosmology in various dimensions [8-13]. One of the intriguing features in these models is that a dynamical compactification of some of the coordinate directions can occur, implying, for example, that a cosmological solution of the ten-dimensional string can evolve so that it describes a four-dimensional expanding universe, with the extra six dimensions undergoing a contraction (see, for example, [11]). In view of the recent advances in the understanding of the unity among string theories, it can be argued that the most natural arenas within which cosmological models should be studied are the type IIA and type IIB string theories, or possibly their M-theory and F-theory progenitors [11]. Cosmological solutions using the R-R fields in type II string theories, as well as those using the NS-NS fields, have been discussed recently in [11]. In this paper, we shall examine a broad class of cosmological models that arise as solutions of the low-energy limits of ten-dimensional string theories or M-theory. Our starting point will be to consider models in the $D$-dimensional toroidal compactifications of the fundamental theories, in which $p$-dimensional spatial sections (which may be flat, spherical or hyperbolic) expand while $q=D-p-1$ dimensions contract, as the universe evolves. These models themselves may all be re-interpreted as ten-dimensional or eleven-dimensional solutions, by reversing the reduction procedure that led to the $D$ dimensional theory. By this means, large classes of ten or eleven dimensional solutions of a cosmological type can be obtained. The extra dimensions that are restored in this oxidation process will themselves typically either expand or contract, adding further to the numbers $p$ and $q$ of expanding and contracting dimensions. Ultimately, phenomenological considerations would imply that one is principally interested in the case where the total number of expanding spatial dimensions is 3 .

The reason for dividing the discussion into two stages, in which first we find cosmological solutions in the $D$-dimensional toroidal compactifications of the ten-dimensional theories, and then we oxidise them back to $D=10$ or $D=11$, is the following. In order to construct solutions, it is useful to choose a highly symmetrical ansatz for the form of the metric and other fields in the theory, so as to obtain relatively simple equations of motion for the remaining degrees of freedom. Upon oxidation to $D=10$ or $D=11$, the solutions typically acquire a more complicated form, which would be less easily found, or classified, 
by a direct ten-dimensional study of the equations of motion. This is analogous to the situation for $p$-brane solitons, where most of the lower-dimensional solutions yield rather complicated configurations in $D=10$ or $D=11$ that correspond to sets of intersecting p-branes, possibly also with "boosts" along certain directions in the toroidally-compactified dimensions [15-20].

The organisation of the paper is as follows. In section 2, we set up the ansatz for the metric tensor and the other fields of the theories, and then obtain cosmological models involving single-scalar, dyonic, and multi-scalar solutions. Included in the multi-scalar cases are models where the equations of motion reduce to the $S L(N+1, R)$ Toda equations. In section 3, we discuss the cosmological properties of some of the models. In section 4, we discuss the Kaluza-Klein reduction and oxidation of the cosmological solutions. The paper ends with conclusions in section 5 .

\section{Cosmological solutions from string theories}

Cosmological models are described by solutions of the low-energy effective theory in which the metric tensor, and the other fields, are time dependent. We shall look for solutions in which the $D$-dimensional metric takes the form

$$
d s^{2}=-e^{2 U} d t^{2}+e^{2 A} d \bar{s}^{2}+e^{2 B} d y^{m} d y^{m}
$$

where the functions $U, A$ and $B$ depend only on $t$, and $d \bar{s}^{2}$ represents the $p$-dimensional metric on the spatial section of a $d$-dimensional spacetime, with $d=p+1$. Typically, we shall consider spatial metrics of the maximally-symmetric form

$$
d \bar{s}^{2}=\frac{d r^{2}}{1-k r^{2}}+r^{2} d \Omega^{2}
$$

where $d \Omega^{2}$ is the metric on a unit $(d-2)$-sphere. Without loss of generality, the constant $k$ may be taken to be equal to 0,1 or -1 , in which case metric $d \bar{s}^{2}$ describes flat, spherical, or hyperboloidal spatial sections respectively. The general idea will be to look for solutions where this spacetime expands at large time, while the $q=D-d$ dimensional space parameterised by the coordinates $y^{m}$ contracts and becomes unobservable at large time. Note that the function $U$ is redundant, in the sense that it can later be set to any desired form by an appropriate redefinition of the time coordinate. It is convenient to include it, however, since the solution of the equations of motion can be simplified by choosing it appropriately.

In the vielbein basis $e^{0}=e^{U} d t, e^{a}=e^{A} \bar{e}^{a}, e^{m}=e^{B} d y^{m}$ (there will be no confusion between the exponential functions and the vielbeins), we find that the curvature 2 -forms 
are given by

$$
\begin{aligned}
\Theta^{0}{ }_{a} & =e^{-2 U}\left(\ddot{A}-\dot{U} \dot{A}+\dot{A}^{2}\right) e^{0} \wedge e^{a}, \\
\Theta^{0}{ }_{m} & =e^{-2 U}\left(\ddot{B}-\dot{U} \dot{B}+\dot{B}^{2}\right) e^{0} \wedge e^{m}, \\
\Theta^{a}{ }_{b} & =\bar{\Theta}^{a}{ }_{b}+e^{-2 U} \dot{A}^{2} e^{a} \wedge e^{b}, \\
\Theta^{a}{ }_{m} & =e^{-2 U} \dot{A} \dot{B} e^{a} \wedge e^{m}, \\
\Theta^{m}{ }_{n} & =e^{-2 U} \dot{B}^{2} e^{m} \wedge e^{n},
\end{aligned}
$$

where a dot denote a derivative with respect to the time coordinate $t$, and $\bar{\Theta}^{a}{ }_{b}$ is the curvature 2-form for the metric $d \bar{s}^{2}=\bar{e}^{a} \bar{e}^{a}$ of the spatial sections, in the vielbein basis $\bar{e}^{a}$. It follows that the tangent-space components of the Ricci tensor for the metric (1) are given by

$$
\begin{aligned}
R_{00} & =-e^{-2 U}\left(p\left(\ddot{A}+\dot{A}^{2}-\dot{U} \dot{A}\right)+q\left(\ddot{B}+\dot{B}^{2}-\dot{U} \dot{B}\right)\right) \\
R_{a b} & =e^{-2 U}\left(\ddot{A}+p \dot{A}^{2}-\dot{U} \dot{A}+q \dot{A} \dot{B}\right) \delta_{a b}+e^{-2 A} \bar{R}_{a b}, \\
R_{m n} & =e^{-2 U}\left(\ddot{B}+q \dot{B}^{2}-\dot{U} \dot{B}+p \dot{A} \dot{B}\right) \delta_{m n}
\end{aligned}
$$

where $\bar{R}_{a b}$ denotes the tangent-space components of the Ricci tensor for the spatial metric. In all the cases we shall consider, the metric $d \bar{s}^{2}$ is Einstein, and we may write $\bar{R}_{a b}=$ $k(p-1) \delta_{a b}$, which is in particular the case for the metrics (2).

In the rest of this section, we shall derive various classes of cosmological solutions in toroidally-compactified string theories.

\subsection{One-scalar cosmological solutions}

The simplest cosmological solution in $D$ dimensions involves the metric, a dilaton and an $n$-rank antisymmetric field strength $F_{n}$. The Lagrangian is given by

$$
e^{-1} \mathcal{L}=R-\frac{1}{2}(\partial \phi)^{2}-\frac{1}{2 n !} e^{a \phi} F_{n}^{2},
$$

where the constant $a$ can be parameterised as [21]

$$
a^{2}=\Delta-\frac{2(n-1)(D-n-1)}{D-2} .
$$

(Note that the Brans-Dicke Lagrangian generalised to include a field strength can be recast in the form (5), by making a dilaton-dependent conformal rescaling of the metric, and scaling the dilaton by an appropriate constant. The Brans-Dicke parameter $\omega$ is related to the dilaton coupling constant a.) In supergravity theories, the full bosonic Lagrangian can 
be consistently truncated to the single-scalar lagrangian (5) for $\Delta=4 / N$, where $N$ is a set of integers $1,2, \ldots, N_{\max }$, where $N_{\max }$ depends on $D$ and $n$ [22]. For 1-form field strengths, the Lagrangian can also be consistently truncated to a set of $N$ 1-forms for which $\Delta$ takes the values $24 /(N(N+1)(N+2))$ [23]. The equations of motion from the Lagrangian (5) are

$$
\begin{aligned}
\square \phi & =\frac{a}{2 n !} e^{a \phi} F^{2}, \\
R_{M N} & =\frac{1}{2} \partial_{M} \phi \partial_{N} \phi+S_{M N}, \\
\partial_{M_{1}}\left(e e^{a \phi} F^{M_{1} \cdots M_{n}}\right) & =0
\end{aligned}
$$

where $S_{M N}$ is a symmetric tensor given by

$$
S_{M N}=\frac{1}{2(n-1) !} e^{a \phi}\left(F_{M N}^{2}-\frac{n-1}{n(D-1)} F^{2} g_{M N}\right)
$$

There are two types of ansätze for the field strength $F_{n}$ that are compatible with the symmetries of the metric (11), giving rise to elementary and solitonic cosmological solutions. In the elementary solutions, the ansatz for the antisymmetric tensor is given in terms of its potential, and in a coordinate frame takes the form

$$
A_{m_{1} i_{2} \cdots m_{q}}=f \epsilon_{m_{1} m_{2} \cdots m_{q}}
$$

and hence

$$
F_{0 m_{1} m_{2} \cdots m_{q}}=\dot{f} \epsilon_{m_{1} m_{2} \cdots m_{q}}
$$

where $f$ is a function of $t$ only. Here and throughout this paper $\epsilon_{M \cdots N}$ and $\epsilon^{M \cdots N}$ are taken to be tensor densities of weights -1 and 1 respectively, with purely numerical components \pm 1 or 0 . Note in particular that they are not related just by raising and lowering indices using the metric tensor. For elementary solutions, we have $p=D-n$ and $q=n-1$.

For the solitonic cosmological solutions, the ansatz for the tangent-space components for the antisymmetric tensor is

$$
F_{a_{1} a_{2} \cdots a_{p}}=\lambda e^{-p A} \epsilon_{a_{1} a_{2} \cdots a_{p}}
$$

where $\lambda$ is a constant. Thus we have $p=n$ and $q=D-n-1$. The form of the exponential prefactor is determined by the requirement that $F_{n}$ satisfy the Bianchi identity $d F_{n}=0$.

Substituting the ansätze for the metric and the field strength into the equations of motion (7), we find

$$
\ddot{\phi}+(p \dot{A}+q \dot{B}-\dot{U}) \dot{\phi}=\frac{1}{2} \epsilon a \lambda^{2} e^{-\epsilon a \phi-2 p A+2 U},
$$




$$
\begin{aligned}
\ddot{A}+(p \dot{A}+q \dot{B}-\dot{U}) \dot{A}+k(p-1) e^{2 U-2 A} & =\frac{q}{2(D-2)} \lambda^{2} e^{-\epsilon a \phi-2 p A+2 U}, \\
\ddot{B}+(p \dot{A}+q \dot{B}-\dot{U}) \dot{B} & =-\frac{p-1}{2(D-2)} \lambda^{2} e^{-\epsilon a \phi-2 p A+2 U}, \\
p\left(\ddot{A}+\dot{A}^{2}-\dot{U} \dot{A}\right)+q\left(\ddot{B}+\dot{B}^{2}-\dot{U} \dot{B}\right)+\frac{1}{2} \dot{\phi}^{2} & =-\frac{p-1}{2(D-2)} \lambda^{2} e^{-\epsilon a \phi-2 p A+2 U},
\end{aligned}
$$

where $\epsilon=1$ for the elementary case and $\epsilon=-1$ for the solitonic case. In the elementary case, the constant $\lambda$ arises as the integration constant for the function $f$ in (9).

It is convenient to make the gauge choice $U=p A+q B$, and to define

$$
X \equiv q B+(p-1) A, \quad Y \equiv B+\frac{(p-1)}{\epsilon a(D-2)} \phi, \quad \Phi \equiv-\epsilon a \phi+2 q B .
$$

The equations of motion for $X, \Phi$ and $Y$ become

$$
\ddot{X}+k(p-1)^{2} e^{2 X}=0, \quad \ddot{\Phi}+\frac{1}{2} \Delta \lambda^{2} e^{\Phi}=0, \quad \ddot{Y}=0,
$$

together with the first integral

$$
\dot{\Phi}^{2}+\Delta \lambda^{2} e^{\Phi}+\frac{2 q(D-2) a^{2}}{p-1} \dot{Y}^{2}=\frac{2 p \Delta}{p-1}\left(\dot{X}^{2}+k(p-1)^{2} e^{2 X}\right) .
$$

Thus $X$ and $\Phi$ both satisfy Liouville equations. The manifest positivity of the left-hand side of (15) shows that the Hamiltonian $\dot{X}^{2}+k(p-1)^{2} e^{2 X}$ for $X$ must be positive, and hence the appropriate form of the solution is

$$
\begin{aligned}
e^{-X} & = \begin{cases}\frac{p-1}{c} \cosh (c t+\delta), & \text { if } k=1 ; \\
\frac{p-1}{c} \sinh (c t+\delta), & \text { if } k=-1 ;\end{cases} \\
X & =-c t-\delta, \quad \text { if } k=0,
\end{aligned}
$$

where $c$ and $\delta$ are constants. Note that in taking the square root of $e^{2 X}$, the positive root should be chosen in the expression for $e^{-X}$. The Hamiltonian $\dot{\Phi}^{2}+\Delta \lambda^{2} e^{\Phi}$ for $\Phi$ is also manifestly positive, and so the solution can be written as

$$
e^{-\frac{1}{2} \Phi}=\frac{\lambda \sqrt{\Delta}}{2 \beta} \cosh (\beta t+\gamma),
$$

where $\beta$ and $\gamma$ are constants. The solution for $Y$ may be taken to be simply

$$
Y=-\mu t
$$

The constraint (15) therefore implies that

$$
\beta^{2}=\frac{p \Delta c^{2}-q(D-2) a^{2} \mu^{2}}{2(p-1)} .
$$


In terms of the original functions $A, B$ and $U$ appearing in the metric (11), and the dilaton $\phi$, the solution takes the form

$$
\begin{aligned}
e^{\frac{\Delta(D-2)}{2 q} A} & =\frac{\lambda \sqrt{\Delta}}{2 \beta} \cosh (\beta t+\gamma) e^{\frac{a^{2}(D-2) \mu t}{2(p-1)}} e^{\frac{\Delta(D-2)}{2 q(p-1)} X} \\
e^{-\frac{\Delta(D-2)}{2(p-1)} B} & =\frac{\lambda \sqrt{\Delta}}{2 \beta} \cosh (\beta t+\gamma) e^{\frac{a^{2}(D-2) \mu t}{2(p-1)}} \\
e^{\frac{\Delta}{2 \epsilon a} \phi} & =\frac{\lambda \sqrt{\Delta}}{2 \beta} \cosh (\beta t+\gamma) e^{-\mu q t}
\end{aligned}
$$

together with $U=p A+q B$. In a case where there is no dilaton, the solutions for $A$ and $B$ are again given by (20), with $\mu=0$. If instead $q=0$, we have from (6) that $a^{2}=\Delta$; the solution for $\phi$ follows from (20) by setting $q=0$, and $A$ is given by $A=X /(p-1)$, with $X$ given by (16).

\subsection{Dyonic cosmological solutions}

In general dimensions, the cosmological solutions are either elementary or solitonic. In $D=2 n$, the $n$-rank field strength can carry both electric (10) and magnetic (11) charges.

In this case, $p=n$ and $q=n-1$. Making the same gauge choice $U=n A+(n-1) B$, we find that the equations of motion are given by

$$
\begin{aligned}
& \ddot{\phi}=\frac{1}{2} a\left(\lambda_{1}^{2} e^{-a \phi}-\lambda_{2}^{2} e^{a \phi}\right) e^{2(n-1) B}, \\
& \ddot{A}+k(n-1) e^{2(n-1)(A+B)}=\frac{1}{4}\left(\lambda_{1}^{2} e^{-a \phi}+\lambda_{2}^{2} e^{a \phi}\right) e^{2(n-1) B}, \\
& \ddot{B}=-\frac{1}{4}\left(\lambda_{1}^{2} e^{-a \phi}+\lambda_{2}^{2} e^{a \phi}\right) e^{2(n-1) B}, \\
& p\left(\ddot{A}+\dot{A}^{2}-\dot{U} \dot{A}\right)+q\left(\ddot{B}+\dot{B}^{2}-\dot{U} \dot{B}\right)+\frac{1}{2} \dot{\phi}^{2}=-\frac{1}{4}\left(\lambda_{1}^{2} e^{-a \phi}+\lambda_{2}^{2} e^{a \phi}\right) e^{2(n-1) B} .
\end{aligned}
$$

These equations can be simplified by defining the variables $X, q_{1}$ and $q_{2}$ :

$$
\begin{aligned}
X & =(n-1)(A+B) \\
B & =\frac{1}{4(n-1)}\left(q_{2}+q_{1}-2 \log \left((n-1) \lambda_{1} \lambda_{2}\right)\right), \\
\phi & =\frac{a}{2(n-1)}\left(q_{2}-q_{1}\right)+\frac{1}{a} \log \frac{\lambda_{1}}{\lambda_{2}},
\end{aligned}
$$

leading to

$$
\ddot{X}+k(n-1)^{2} e^{2 X}=0, \quad \ddot{q}_{1}=-e^{\alpha q_{1}+(1-\alpha) q_{2}}, \quad \ddot{q}_{2}=-e^{(1-\alpha) q_{1}+\alpha q_{2}},
$$

together with the first-order constraint

$$
\frac{1}{2} \alpha\left(\dot{q}_{1}^{2}+\dot{q}_{2}^{2}\right)+(1-\alpha) \dot{q}_{1} \dot{q}_{2}+e^{\alpha q_{1}+(1-\alpha) q_{2}}+e^{\alpha q_{2}+(1-\alpha) q_{1}}=2 n\left(\dot{X}^{2}+k(n-1)^{2} e^{2 X}\right) .
$$


Here the constant $\alpha$ is given by

$$
\alpha=\frac{1}{2}+\frac{a^{2}}{2(n-1)}=\frac{\Delta}{2(n-1)} .
$$

The solution for $X$ is straightforward, and is given by (16), with $p=n$. For generic values of $\alpha$, a particular solution for $q_{1}$ and $q_{2}$ can be obtained by setting $q_{1}=q_{2}$, which reduces the equations to two identical Liouville equations. This special solution describes a self-dual cosmological model, with $\lambda_{1}=\lambda_{2}$. It is unclear how to solve the equations in the general dyonic case $\lambda_{1} \neq \lambda_{2}$, for generic values of $\alpha$. However there are two values of $\alpha$ for which the equations are solvable. When $\alpha=1$, they reduce to two Liouville equations and the solutions can be straightforwardly obtained. This value of $\alpha$ can arise for a 3 -form field strength in $D=6$, with $\Delta=4$. Another value of $\alpha$ for which the equations are solvable is $\alpha=2$, in which case they become the $S L(3, R)$ Toda equations. This value of $\alpha$ can arise for a 2-form field strength in $D=4$, with $\Delta=4$. (In fact, this field strength can also support a dyonic black hole solution, whose equations of motion can again be re-expressed as the same $S L(3, R)$ Toda equations [24]. This solution is unstable, in the sense that it is a bound state of an electric and a magnetic black hole with negative binding energy [25].)

\subsection{Multi-charge cosmological solutions}

The $D$-dimensional bosonic Lagrangian of M-theory to $D$ dimensions compactified on a torus can be consistently truncated to

$$
e^{-1} \mathcal{L}=R-\frac{1}{2}(\partial \vec{\phi})^{2}-\frac{1}{2 n !} \sum_{\alpha=1}^{N} e^{\vec{c}_{\alpha} \cdot \vec{\phi}} F_{\alpha}^{2},
$$

when the dilaton vectors for the set of $N$ field strengths $F_{\alpha}$ of rank $n \geq 2$ satisfy the dot products

$$
M_{\alpha \beta}=\vec{c}_{\alpha} \cdot \vec{c}_{\beta}=4 \delta_{\alpha \beta}-\frac{2(n-1)(D-n-1)}{D-2} .
$$

The maximum value $N_{\max }$ for $N$ depends on the rank of the field strengths, and on the dimension $D$. For example for 2-form field strengths, $N_{\max }=2$ for $6 \leq D \leq 9 ; N_{\max }=3$ in $D=5$; and $N_{\max }=4$ in $3 \leq D \leq 4$ [22]. We shall discuss the case of 1 -form field strengths in subsection 2.4. In fact, we can perform a further truncation to the single-scalar Lagrangian (5) with $a, \phi$ and $F$ given by 22]

$$
\begin{aligned}
a^{2} & =\left(\sum_{\alpha, \beta}\left(M^{-1}\right)_{\alpha \beta}\right)^{-1}, \quad \phi=a \sum_{\alpha, \beta}\left(M^{-1}\right)_{\alpha \beta} \vec{c}_{\alpha} \cdot \vec{\phi} \\
F_{\alpha}^{2} & =a^{2} \sum_{\beta}\left(M^{-1}\right)_{\alpha \beta} F^{2} .
\end{aligned}
$$


For dilaton vectors whose dot products satisfy (27), the value of the constant $a$ is given by (6) with $\Delta=4 / N$.

In this subsection, we shall obtain multi-charge cosmological solutions for the Lagrangian (26). We use the same elementary (9) or solitonic (11) ansätze for the field strengths $F_{\alpha}$. The metric ansatz is given by (1), and again we make the gauge choice $U=p A+q B$. The equations of motion become

$$
\begin{aligned}
& \ddot{\vec{\phi}}=\frac{1}{2} \epsilon \sum_{\alpha} \vec{c}_{\alpha} \lambda_{\alpha}^{2} e^{-\epsilon \vec{c}_{\alpha} \cdot \vec{\phi}+2 q B}, \\
& \ddot{A}=-k(p-1) e^{2 q B+2(p-1) A}+\frac{q}{2(D-2)} \sum_{\alpha} \lambda_{\alpha}^{2} e^{-\epsilon \vec{c}_{\alpha} \cdot \vec{\phi}+2 q B}, \\
& \ddot{B}=-\frac{p-1}{2(D-2)} \sum_{\alpha} \lambda_{\alpha}^{2} e^{-\epsilon \vec{c}_{\alpha} \cdot \vec{\phi}+2 q B} . \\
& p\left(\ddot{A}+\dot{A}^{2}-\dot{U} \dot{A}\right)+q\left(\ddot{B}+\dot{B}^{2}-\dot{U} \dot{B}\right)+\frac{1}{2}(\dot{\vec{\phi}})^{2}=-\frac{p-1}{2(D-2)} \sum_{\alpha} \lambda_{\alpha}^{2} e^{-\epsilon \vec{c}_{\alpha} \cdot \vec{\phi}+2 q B} .
\end{aligned}
$$

As in the single-charge case, it is convenient to define new variables:

$$
X \equiv q B+(p-1) A, \quad Y=B+\frac{\epsilon(p-1)}{D-2} \sum_{\alpha, \beta}\left(M^{-1}\right)_{\alpha \beta} \varphi_{\beta}, \quad \Phi_{\alpha}=-\epsilon \varphi_{\alpha}+2 q B,
$$

where $\varphi_{\alpha}=\vec{c}_{\alpha} \cdot \vec{\phi}$. The equations of motion for $X$ and $\Phi_{\alpha}$ become a set of Liouville equations

$$
\ddot{X}+k(p-1)^{2} e^{2 X}=0, \quad \ddot{\Phi}_{\alpha}+2 \lambda_{\alpha}^{2} e^{\Phi_{\alpha}}=0,
$$

together with the first integral constraint

$$
\sum_{\alpha}\left(\dot{\Phi}_{\alpha}^{2}+4 \lambda_{\alpha}^{2} e^{\Phi_{\alpha}}\right)+\frac{8 q(D-2) a^{2}}{(p-1) \Delta} \dot{Y}^{2}=\frac{8 p}{p-1}\left(\dot{X}^{2}+k(p-1)^{2} e^{2 X}\right),
$$

where $\Delta=4 / N$ and $a$ is given by (6), and $Y$ again satisfies $\ddot{Y}=0$.

The solution for the function $X$ depends on the value of the parameter $k$, and is again given by (16). The solutions for $\Phi_{\alpha}$ take the form

$$
e^{-\frac{1}{2} \Phi_{\alpha}}=\frac{\lambda_{\alpha}}{\beta_{a}} \cosh \left(\beta_{\alpha} t+\gamma_{\alpha}\right)
$$

where $\beta_{\alpha}$ and $\gamma_{\alpha}$ are constants. The solution for $Y$ may again be taken to be simply $Y=-\mu t$. The constraint (32) therefore implies that

$$
\sum_{\alpha} \beta_{\alpha}^{2}=\frac{2 p \Delta c^{2}-2 q(D-2) a^{2} \mu^{2}}{(p-1) \Delta} .
$$

In terms of the functions $U, A, B$ and the dilatonic fields $\varphi_{\alpha}$, the solutions can be expressed as

$$
\begin{aligned}
e^{-\frac{2(D-2)}{p-1} B} & =e^{\frac{2(D-2) a^{2} \mu t}{(p-1) \Delta}} \prod_{\alpha=1}^{N}\left(\frac{\lambda_{\alpha}}{\beta_{\alpha}} \cosh \left(\beta_{\alpha} t+\gamma_{\alpha}\right)\right) \\
e^{\frac{2(D-2)}{q} A} & =e^{\frac{2(D-2)}{q(p-1)} X} e^{\frac{2(D-2) a^{2} \mu t}{(p-1) \Delta}} \prod_{\alpha=1}^{N}\left(\frac{\lambda_{\alpha}}{\beta_{\alpha}} \cosh \left(\beta_{\alpha} t+\gamma_{\alpha}\right)\right)
\end{aligned}
$$


with $U=p A+q B$ and $\epsilon \varphi_{\alpha}=2 q B-\Phi_{\alpha}$. When all the parameters $\lambda_{\alpha}$ are equal and all $\beta_{\alpha}$ are equal, multi-charge solutions reduce to the single-scalar solution given by (20)

\section{4 $S L(N+1, R)$ cosmological solutions}

In this subsection, we discuss cosmological solutions with 1-form field strengths. The bosonic Lagrangian of M-theory compactified to $D$ dimensions on a torus can be truncated to one involving $N \leq N_{\max } 1$-form field strengths, of the form (26). Such consistent truncations are possible when the dilaton vectors of the retained field strengths satisfy (27). In this case, we have $N_{\max }=2$ for $7 \leq D \leq 8 ; N_{\max }=4$ for $5 \leq D \leq 6 ; N_{\max }=7$ for $D=4$ and $N_{\max }=8$ for $D=3$.

For the 1-form field strengths, alternative consistent truncations are possible in cases where the dot products of the dilaton vectors do not satisfy (27), but instead satisfy [23]

$$
M_{\alpha \beta}=4 \delta_{\alpha \beta}-2 \delta_{\alpha, \beta+1}-2 \delta_{\alpha, \beta-1}
$$

This is in fact twice the Cartan matrix for $S L(N+1, R)$, and consequently, as we shall see, the equations of motion of the consistently-truncated system (26) can be cast into the form of the $S L(N+1, R)$ Toda equations, which are exactly solvable. The multi-scalar multi-charge system can be further truncated to a single-scalar system (5) using (28); these solutions have 23]

$$
a^{2}=\Delta=\frac{24}{N(N+1)(N+2)} .
$$

It was shown in [23] that sets of dilaton vectors with dot products given by (36) arise in all toroidally-compactified supergravities in all dimensions $D \leq 9$. In this case $N_{\max }=10-D$.

We shall first consider elementary cosmological solutions. The metric for the elementary case involving 1-form field strengths is given by

$$
d s^{2}=-e^{2 U} d t^{2}+e^{2 A} d \bar{s}^{2}
$$

where $d \bar{s}^{2}$ is again the metric on the spatial sections, typically taking the form (2). The field strengths take the elementary form given by (9). It is convenient to make the gauge choice $U=(D-1) A$, which implies that the equations of motion become

$$
\begin{aligned}
& \ddot{\varphi}_{\alpha}=\frac{1}{2} \sum_{\beta} M_{\alpha \beta} \lambda_{\beta}^{2} e^{-\varphi_{\beta}}, \quad \ddot{A}+k(D-2) e^{2(D-2) A}=0, \\
& \sum_{\alpha, \beta}\left(M^{-1}\right)_{\alpha \beta} \dot{\varphi}_{\alpha} \dot{\varphi}_{\beta}+\sum_{\alpha} \lambda_{\alpha}^{2} e^{-\varphi_{\alpha}}=2(D-1)(D-2)\left(\dot{A}^{2}+k e^{2(D-2) A}\right) .
\end{aligned}
$$


Making the further redefinition $\Phi_{\alpha}=-2 \sum_{\beta}\left(M^{-1}\right)_{\alpha \beta} \varphi_{\beta}$, we find that $\Phi_{\alpha}$ satisfies

$$
\begin{aligned}
& \Phi_{\alpha}^{\prime \prime}=-\lambda_{\alpha}^{2} \exp \left(\frac{1}{2} \sum_{\beta} M_{\alpha \beta} \Phi_{\beta}\right) \\
& \frac{1}{4} \sum_{\alpha, \beta} M_{\alpha \beta} \dot{\Phi}_{\alpha} \dot{\Phi}_{\beta}+\sum_{\alpha} \lambda_{\alpha}^{2} \exp \left(\frac{1}{2} \sum_{\beta} M_{\alpha \beta} \Phi_{\beta}\right)=2(D-2)(D-1)\left(\dot{A}^{2}+k e^{2(D-2) A}\right) .
\end{aligned}
$$

Finally, the redefinition $\Phi_{\alpha}=q_{\alpha}-4 \sum_{\beta}\left(M^{-1}\right)_{\alpha \beta} \log \lambda_{\beta}$ removes the charges from the equations, and hence from (36) we see that the equations become

$$
\begin{gathered}
\ddot{q}_{1}=-e^{2 q_{1}-q_{2}}, \\
\ddot{q}_{2}=-e^{-q_{1}+2 q_{2}-q_{3}}, \\
\ddot{q}_{3}=-e^{-q_{2}+2 q_{3}-q_{4}}, \\
\\
\cdots \\
\ddot{q}_{N}=e^{-q_{N-1}+2 q_{N}} .
\end{gathered}
$$

Thus the functions $q_{\alpha}$ satisfy the $S L(N+1, R)$ Toda equations, whilst $A$ satisfies the Liouville equation. The solutions are subject to the first-order constraint in (40), which can be re-expressed as

$$
\mathcal{H}=2(D-1)(D-2)\left(\dot{A}^{2}+k e^{2(D-2) A}\right),
$$

where $\mathcal{H}$ is the Hamiltonian for the Toda equations (41), given by

$$
\mathcal{H}=\frac{1}{4} \sum_{\alpha, \beta} M_{\alpha \beta} \dot{q}_{\alpha} \dot{q}_{\beta}+\sum_{\alpha} \exp \left(\frac{1}{2} \sum_{\beta} M_{\alpha \beta} q_{\beta}\right)
$$

The solution of the Liouville equation for $A$, whose form depends on the value of the constant $k$, is given by (16) with $p=D-1$, where $X=(D-2) A$. The general solution to the $S L(N+1, R)$ Toda equations (41) can be given compactly in the form [26]

$$
e^{-q_{\alpha}}=\sum_{k_{1}<k_{2} \cdots<k_{\alpha}}^{N+1} f_{k_{1}} \cdots f_{k_{\alpha}} \Delta^{2}\left(k_{1}, \ldots, k_{\alpha}\right) e^{\left(\mu_{k_{1}}+\cdots+\mu_{k_{\alpha}}\right) t},
$$

where $\Delta^{2}\left(k_{1}, \cdots, k_{\alpha}\right)=\prod_{k_{i}<k_{j}}\left(\mu_{k_{i}}-\mu_{k_{j}}\right)^{2}$ is the square of the Vandermonde determinant, and $f_{k}$ and $\mu_{k}$ are arbitrary constants satisfying

$$
\prod_{k=1}^{N+1} f_{k}=\Delta^{2}(1,2, \cdots, N+1), \quad \sum_{k=1}^{N+1} \mu_{k}=0 .
$$

The Hamiltonian, which is conserved, takes the value $\mathcal{H}=\frac{1}{2} \sum_{\alpha=1}^{N+1} \mu_{\alpha}^{2}$. It follows from (42) that the Hamiltonian constraint implies

$$
\sum_{k=1}^{N+1} \mu_{k}^{2}=4(D-1)(D-2) c^{2}
$$


It is straightforward to generalise the above discussion to solitonic cosmological solutions, where we have $p=1$ and $q=D-2$. The equations of motion again can be cast into the form of $S L(N+1, R)$ Toda equations, and hence we can obtain exact solutions.

\section{Cosmological characteristics of the solutions}

The solutions that we have obtained in the previous section have metrics of the form (1) in which the scale factors $e^{A}$ and $e^{B}$ evolve in time. In order to obtain realistic cosmological models, a first requirement is that the scale factor $e^{A}$ for the spatial sections of the universe should evolve from a small value at early times to a large value at late times. Furthermore, one would hope that the scale factor $e^{B}$ for the additional $q=D-p-1$ dimensions parameterised by the $y^{m}$ coordinates would become small at large times, so that the additional dimensions become unobservable.

In order to discuss the evolution of the solutions, it is useful to introduce a comoving time coordinate. In cases where there is no dilaton, such as solutions of $D=11$ supergravity or M-theory, the choice of metric is unambiguous, and the comoving time $\tau$ is given by $\tau=\int e^{U} d t$. In cases where there is a dilaton, such as solutions of the type IIA string in $D=10$, there are two natural metrics that one might consider, namely the Einstein-frame metric and the string-frame metric. The former is the one that we have been considering thus far in the paper, and the latter is related to it by the conformal rescaling $d s_{\text {string }}^{2}=e^{\phi} d s^{2}$. The comoving time coordinate depends on the choice of metric.

We shall begin by considering the simpler case of solutions in $D=11$ supergravity, and then afterwards we shall consider solutions in $D=10$.

\subsection{Cosmology in $D=11$}

In $D=11$ supergravity, the bosonic fields consist only of the metric and a 4 -form field strength. We can use it to construct both elementary cosmological solutions with $p=7$ and solitonic solutions with $p=4$. Their metrics are given by the first two equations in (20), with $\Delta=4$ and $\mu=0$, owing to the absence of the dilaton. We may also take $\gamma=0$, by appropriate choice of the origin for $t$. Let us consider the solitonic case, which will

correspond to a 5 -dimensional cosmological model. We see from (19) that $\beta^{2}=\frac{8}{3} c^{2}$ in this case, and from (20) that

$$
e^{3 A}=\frac{\lambda}{\beta} e^{X} \cosh \beta t
$$




$$
e^{-6 B}=\frac{\lambda}{\beta} \cosh \beta t
$$

and $U=4 A+6 B$. Without loss of generality, we may take $\beta$ to be positive. The scale factor $R \equiv e^{A}$ diverges both at $t=\infty$ and at $t=-\infty$, since $\beta>|c|$, while the scale factor $e^{B}$ associated with the extra $q$ dimensions tends to zero in both limits. The comoving time $\tau$, defined such that $d s^{2}=-d \tau^{2}+e^{2 A} d \bar{s}^{2}+e^{2 B} d y^{m} d y^{m}$, is given by $\tau=\int e^{U} d t$. In general, owing to the complexity of the function $e^{U}$, the relation between $\tau$ and $t$ can only be evaluated by numerical methods.

If $k=1$, the comoving coordinate $\tau$ is finite for all values of $t$. When $t$ runs from $-\infty$ to $+\infty, \tau$ runs between two finite values, $\tau_{-}$to $\tau_{+}$. In this process, the scale size $R=e^{A}$ shrinks from infinite size at $\tau_{-}$to a minimum at some value $\tau_{0}$ and then expands again to infinity at $\tau_{+}$. From (3), and the form of the solution (47), we see that the curvature is of order $e^{-2 U}$ at large $|t|$, and thus diverges as $\exp \left(\left(\frac{8}{3}-\frac{2}{3} \sqrt{\frac{8}{3}}\right)|c t|\right)$. This solution is obviously undesirable from both the phenomenological and the theoretical points of view. The situation is different when $k=0$, since then we have $e^{U} \sim \exp \left(\frac{1}{3}(\beta|t|-4 c t)\right)$ at large $|t|$. If $c$ is negative, $c=-\sqrt{\frac{3}{8}} \beta$, then $\tau$ diverges as $t$ tends to infinity, and in fact $\tau \sim e^{U}$. Since $e^{-2 U}$ goes to zero at large $\tau$, it follows that the curvature goes to zero at large $\tau$. Finally, if $k=-1$ the coordinate $t$ runs from $-\delta / c$ to $\pm \infty$, and $\tau$ correspondingly runs from infinity to zero. The scale size $R$ becomes large as $\tau$ tends to infinity, and is zero when $\tau$ is zero. The curvature is singular at $\tau=0$, and tends to zero as $\tau$ tends to infinity. Thus both the $k=0$ and $k=-1$ models have the feature that the universe expands as the comoving time increases from some finite time $\tau_{0}$ to infinity, i.e. $R^{\prime}>0$ for $\tau>\tau_{0}$, where a prime denotes a derivative with respect to $\tau$. One may define $\tau_{0}$, where the scale factor $R$ is a minimum, as the starting point for the expansion of the universe. In the $k=0$ case, we have $R^{\prime \prime}>0$ at $\tau=\tau_{0}$ and $R^{\prime \prime}<0$ as $\tau \rightarrow \infty$. When $k=-1$, we have $R^{\prime \prime}>0$ at $\tau=\tau_{0}$, and $R^{\prime \prime}=0$ as $\tau$ tends to infinity. In these $k=0$ and $k=-1$ solutions, the universe is not starting from zero size at $\tau=\tau_{0}$, but rather, this value of the comoving time represents the point at which it has a minimum size, which is of the order of the Planck scale. In principle, one can extrapolate back to $\tau=0$, at which point the scale size $R$ is infinite. Although this region $0 \leq \tau \leq \tau_{0}$ does not itself describe a satisfactory cosmological evolution, since the comoving time reaches an endpoint at $\tau=0$ and the curvature diverges there, there is a sense in which one can think of the "physical" universe with $\tau \geq \tau_{0}$ as emerging through a wormhole at $\tau=\tau_{0}$.

In a similar manner, one can analyse the elementary solutions in $D=11$, which describe an 8-dimensional cosmological model. 


\subsection{Cosmology in $D=10$}

Since ten-dimensional string theories have 3-form field strengths, we can obtain solitonic solutions with $p=3$ and $q=6$, which describe 4-dimensional cosmological models. The dilaton coupling is such that $a^{2}=1$, and hence $\Delta=4$. The string coupling constant is given by $g=e^{-\phi}$. The NS-NS 3-form field strengths of any of the $D=10$ string theories have dilaton coupling $a=+1$, while the R-R 3 -form of the type IIB theory has $a=-1$. From (20), we find that the solutions have the form

$$
\begin{aligned}
e^{\frac{8}{3} A} & =\frac{\lambda}{\beta} \cosh (\beta t+\gamma) e^{2 \mu t+\frac{4}{3} X}, \\
e^{-8 B} & =\frac{\lambda}{\beta} \cosh (\beta t+\gamma) e^{2 \mu t}, \\
e^{-\frac{2}{a} \phi} & =\frac{\lambda}{\beta} \cosh (\beta t+\gamma) e^{-6 \mu t},
\end{aligned}
$$

together with $U=3 A+6 B$, and from (19) we have $\beta^{2}=3 c^{2}-12 \mu^{2}$.

Let us first consider the case when $\mu=0$. This value has the distinguishing feature that the dimensional reduction of the solution by compactifying the $y^{m}$ coordinates gives rise to solutions which also involve only one scalar field, as we shall discuss in section 4 . In the Einstein frame, the analysis of the cosmological properties of these solutions is analogous to that for $D=11$. When $k=1$, the comoving coordinate $\tau$ runs from $\tau_{-}$to $\tau_{+}$as $t$ runs from $-\infty$ to $+\infty$. The 4 -dimensional scale size shrinks from infinity to a minimum and then expands to infinity again. For the case $k=0$, and $c<0$, the comoving coordinate runs from zero to infinity as $t$ runs from $-\infty$ to $\infty$. The scale parameter $R=e^{A}$ diverges in both the $\tau \rightarrow 0$ and $\tau \rightarrow \infty$ regimes. Thus we can define $\tau_{0}$, at which the scale parameter $R$ is a minimum, as the starting point of the expansion of the universe, with $\tau$ running from $\tau_{0}$ to infinity. It is easy to verify that speed of the expansion $R^{\prime}$ is always greater than zero when $\tau>\tau_{0}$, but with $R^{\prime \prime}>0$ when $\tau \rightarrow \tau_{0}$ and $R^{\prime \prime}<0$ when $\tau \rightarrow \infty$. Although the metrics behave identically for both the NS-NS and R-R solutions, the dilaton field, and hence the string coupling $g$, behave in opposite ways. For the NS-NS solution, the string coupling diverges when $\tau \rightarrow \infty$, whilst for the R-R solution the string coupling vanishes in that limit. In all cases, the curvature tends to zero when the scale factor $R$ is large, if this coincides with $\tau$ going to infinity, namely in the $k=0$ and $k=-1$ models. On the other hand, if large $R$ corresponds to a finite value of $\tau$, as in the $k=1$ models, the curvature diverges there.

If $k=-1$, the coordinate $t$ runs from $\pm \infty$ to $t=-\delta / c$, and correspondingly, the comoving coordinate $\tau$ runs from zero to infinity. The scale parameter $R$ runs from infinity 
to a minimum at $\tau_{0}$, and then to infinity again. Thus we can define the expansion of the universe from $\tau=\tau_{0}$ to $\tau=\infty$. At the beginning of the universe, we have $R^{\prime \prime}>0$ whilst at the end of universe we have $R^{\prime \prime}=0$ in this case. At the beginning $\tau=\tau_{0}$, the string coupling constant $g$ is a non-vanishing constant for both NS-NS solutions and R-R solutions and it converges to another non-vanishing constant at large $\tau$.

In the above discussion, we studied the cosmological characteristics of the metrics in the Einstein frame. In this frame, the form of the metrics is insensitive to whether the solution is constructed using an NS-NS 3-form or an R-R 3-form. However, since the constant $a$ in the dilaton prefactor is +1 for the NS-NS solutions and -1 for the R-R solutions, the string metrics for the NS-NS and R-R cases are quite different, and we shall discuss them separately. In the string-frame metric, the scale factor $e^{B}$ for the $y^{m}$ space in the R-R solutions diverges when $|t|$ goes to infinity. Thus the solutions only make sense when $k=-1$, since in this case the relevant part of the evolution does not involve the large $|t|$ regime. Specifically, as $t$ runs from $\pm \infty$ to $-\delta / c$, the comoving coordinate $\tau$ runs from zero to infinity, but the evolution is taken from $\tau=\tau_{0}$ where $R=e^{A}$ is a minimum to $\tau=\infty$ where $R$ diverges. This behaviour of the scale factor $R$ is similar to that in the Einstein frame, but the scale factor $e^{B}$ shrinks, although remaining finite and non-zero for the entire evolution. For the NS-NS solutions, the string coupling diverges at large $|t|$. Thus the solutions are again restricted to the case $k=-1$, where $t$ runs from $\pm \infty$ to $t=-\delta / c$. The comoving coordinate $\tau$ runs from zero to infinity. At large $\tau$, the $D=4$ universe expands with constant speed; but at $\tau=0$, unlike in the Einstein frame, we have $R=e^{A}=0$, and $R^{\prime \prime}<0$ for small $\tau$.

So far, we have discussed the cosmological characteristics of the solution (48) when $\mu$ is set to zero. We saw that the string coupling diverges for NS-NS solutions at large $|t|$, while it vanishes for R-R solutions. Now let us examine the solutions when $\mu$ is non-vanishing. In this case, as we shall see in the next section, dimensional reductions of the solutions in which the $y^{m}$ coordinates are compactified give rise to solutions with additional scalar fields in the lower dimension. We shall examine the metrics for solutions with $\beta=6 \mu>0$. In this case the dilaton, and hence the string coupling, becomes a constant when $t$ goes to infinity, for both the NS-NS and the R-R solutions. Then, by making a small perturbation away from $\beta=6 \mu$, which will not qualitatively affect the characteristics of the metric, we can have the string coupling vanish when $t$ goes to infinity. It follows from the equation below (48) that we have $|c|=4 \mu$ when $\beta=6 \mu$. In the Einstein frame, the behaviour of the solutions for $k=1, k=0$ and $k=-1$ are analogous to the corresponding ones 
with $\mu=0$ that we discussed previously. This implies, in particular, that by adjusting the parameter $\mu$ properly, we can have an inflationary model even for NS-NS solutions when $k=0$, where the string coupling vanishes as the comoving time approaches infinity. At large $t$ the behaviour of the metric in the string frame is the same as that in the Einstein frame, since the dilaton tends to a constant at large $t$. If $k=0$, large values of $t$ imply large values of $\tau$, whilst if $k=-1$, they imply that $\tau$ tends to zero. Thus in the string frame, by adjusting the parameter $\mu$ properly, we can also have inflationary models where the string coupling respectively vanishes or goes to a constant at large $\tau$, while the expansion rate $R^{\prime}$ of the 4-dimensional universe either tends to zero or becomes a constant.

We have discussed the cosmological features of the solitonic solutions for both the NSNS and R-R 3-forms. These solutions can provide inflationary models of the universe. The 9-dimensional space divides into two parts: a 6-dimensional subspace shrinks to zero or a finite size as the comoving time tends to infinity, while a 3-dimensional subspace expands. The solutions provide a dynamical compactification of the 10-dimensional spacetime to $D=4$.

In $D=10$, there exist further field strengths of other ranks, and the associated solutions will describe cosmologies in different dimensions.

\section{Dimensional reduction and oxidation of cosmological solu- tions}

In the previous sections, we constructed rather general classes of cosmological solutions in $D$-dimensional supergravity theories. Ultimately, one views these theories as originating from some fundamental theory such a string in $D=10$, or M-theory in $D=11$. Since the lower-dimensional theories that we have considered are obtained by consistent dimensional reduction from $D=10$ or $D=11$, it follows that all their cosmological solutions can be oxidised back to solutions in the fundamental higher dimension. In part, the utility of constructing solutions first in the lower dimension is that it can often be simpler than solving the equations directly in the fundamental dimension. In particular, this is true if the lower-dimensional solution involves more than one field strength, since its oxidation to the higher dimension will then give a solution that lies outside the class that we have considered thus far. Thus it is useful to study the general procedure of oxidation and reduction of the various cosmological solutions. 


\subsection{Kaluza-Klein dimensional reduction}

The general procedure of toroidal dimensional reduction to can be broken down into a sequence of one-step reductions on circles. The necessary reduction formulae can thus be encapsulated in the reduction of the following Lagrangian in $(D+1)$ dimensions,

$$
\mathcal{L}_{D+1}=\hat{e} \hat{R}-\frac{1}{2} \hat{e}(\partial \hat{\phi})^{2}-\frac{e}{2 n !} e^{\hat{a} \hat{\phi}} \hat{F}_{n}^{2},
$$

giving

$$
\begin{aligned}
\mathcal{L}_{D}= & e R-\frac{1}{2} e(\partial \phi)^{2}--\frac{1}{2} e(\partial \varphi)^{2}-\frac{1}{4} e e^{-2(D-1) \alpha \varphi} \mathcal{F}^{2} \\
& -\frac{e}{2 n !} e^{-2(n-1) \alpha \varphi-\hat{a} \phi}{F_{n}^{\prime}}^{2}-\frac{e}{2(n-1) !} e^{2(D-n) \alpha \varphi-\hat{a} \phi} F_{n-1}^{2},
\end{aligned}
$$

in $D$ dimensions. The $(D+1)$-dimensional hatted fields are expressed in terms of the $D$-dimensional unhatted fields by the standard Kaluza-Klein relations:

$$
\begin{aligned}
& d \hat{s}_{D+1}^{2}=e^{2 \alpha \varphi} d s_{D}^{2}+e^{-2(D-2) \alpha \varphi}(d z+\mathcal{A})^{2}, \\
& \hat{A}_{n-1}=A_{n-1}+A_{n-2} \wedge d z, \quad \hat{\phi}=\phi,
\end{aligned}
$$

where all the unhatted fields are independent of the compactification coordinate $z$, and $\mathcal{F}=d \mathcal{A}$. The constant $\alpha$ is given by $\alpha=(2(D-1)(D-2))^{-1 / 2}$. The lower-dimensional field strengths, obtained from the exterior derivative of the expression for the potential $\hat{A}_{n-1}$ given above, are therefore expressed as $\hat{F}_{n}=F_{n}^{\prime}+F_{n-1} \wedge(d z+\mathcal{A})$, where $F_{n}^{\prime}$ is the Chern-simons corrected form $F_{n}^{\prime}=d A_{n-1}-d A_{n-2} \wedge \mathcal{A}$, and $F_{n-1}=d A_{n-2}$.

Let us begin by applying the above formalism to the example of a $D=10$ cosmological solution, of the form (20) with $p=3$ and $q=6$, with $\Delta=4$ and $a^{2}=1$. We shall take the compactification coordinate $z$ to be one of the $y^{m}$ coordinates, so that in $D=9$ we have $p=3$ and $q=5$. The relevant part of the $D=9$ Lagrangian will be, using (50),

$$
\begin{aligned}
\mathcal{L}_{9} & =e R-\frac{1}{2} e(\partial \phi)^{2}-\frac{1}{2} e(\partial \varphi)^{2}-\frac{1}{12} e e^{a \phi-4 \alpha \varphi} F_{3}^{2} \\
& =e R-\frac{1}{2} e\left(\partial \phi_{1}\right)^{2}-\frac{1}{2} e\left(\partial \phi_{2}\right)^{2}-\frac{1}{12} e e^{b \phi_{1}} F_{3}^{2}
\end{aligned}
$$

where in the second line we have introduced a rotated pair of dilatonic scalars, defined by $b \phi_{1}=a \phi-4 \alpha \varphi$ and $b \phi_{2}=4 \alpha \phi+a \varphi$. The constants $\alpha$ and $b$ are given by $\alpha=1 /(4 \sqrt{7})$ and $b^{2}=8 / 7$. Comparing the metric $d s_{10}^{2}$ given in (1) for the ten-dimensional solution with the dimensionally-reduced metric $d s_{9}^{2}$ defined in (51), we see that the Kaluza-Klein scalar $\varphi$ is given by $\alpha \varphi=-B / 7$. Thus it follows from the ten-dimensional solution (20) that its 
dimensional reduction to $D=9$ gives a metric of the form (11), with functions $\tilde{U}, \tilde{B}$ and $\tilde{B}$ (the tildes denoting $D=9$ quantities) given by

$$
\begin{aligned}
e^{\frac{14}{5} \tilde{A}} & =\frac{\lambda}{\beta} \cosh (\beta t+\gamma) e^{2 \mu t+\frac{7}{5} X}, \\
e^{-7 \tilde{B}} & =\frac{\lambda}{\beta} \cosh (\beta t+\gamma) e^{2 \mu t},
\end{aligned}
$$

together with $\tilde{U}=2 \tilde{A}+5 \tilde{B}$. The $D=9$ dilatonic fields $\phi_{1}$ and $\phi_{2}$ are given by

$$
\begin{aligned}
e^{-\frac{2}{b} \phi_{1}} & =\frac{\lambda}{\beta} \cosh (\beta t+\gamma) e^{-5 \mu t}, \\
e^{-\frac{4 \sqrt{2}}{b} \phi_{2}} & =e^{-8 \mu t} .
\end{aligned}
$$

Thus we see that in general, the dimensional reduction of a single-scalar solution has given rise to a solution with two linearly-independent scalars. If, however, we consider the $D=10$ solution with $\mu=0$, then it reduces to a single-scalar solution in $D=9$. In fact this $D=9$ solution is precisely of the same form (20), with $p=3, q=5, \Delta=4$ and $\mu=0$. It is interesting to note that even when $\mu$ is taken to be non-zero, the $D=9$ metric is still of the form given in (20). However, the Hamiltonian relation (19) between the integration constants $\beta, c$ and $\mu$ is given by the ten-dimensional formula $\beta^{2}=3 c^{2}-12 \mu^{2}$ rather than the nine-dimensional formula $\beta^{2}=3 c^{2}-10 \mu^{2}$ that would be needed if the $\mu \neq 0$ solutions were to have the single-scalar form (20) in $D=9$. Thus the reason why the additional scalar $\phi_{2}$ is excited in the $\mu \neq 0$ dimensionally-reduced solutions is that its energy contribution is needed in order to make up the deficit in the Hamiltonian constraint.

It is interesting to note that the dimensional reduction of the 10-dimensional cosmological solutions gives rise to new solutions that are beyond the scope of section 2 , in that an additional scalar, namely $\phi_{2}$, which does not couple to the field strength, becomes linearly proportional to the time coordinate $t$. The metric and the dilaton $\phi_{1}$ of the solution, however, have exactly the same form as those for the solution with vanishing $\phi_{2}$. In fact the constant of proportionality $\nu$ in the time dependence of $\phi_{2}=\nu t$ can be arbitrary, and has the effect of changing the relation between the constants of integration to $\beta^{2}=3 c^{2}-10 \mu^{2}-\nu^{2}$, where $\nu$ is the contribution from $\phi_{2}$. These $D=9$ solutions can also be oxidised to $D=10$, but for generic values of $\nu$ the internal coordinate $z$ can no longer be isotropically grouped with the coordinates $y^{m}$. Thus these $D=9$ solutions gives rise to new ten-dimensional cosmological solutions, but in this case the $y^{m}$ and $z$ spaces dynamically compactify at different rates.

The above illustration of Kaluza-Klein dimensional reduction of the $D=10$ cosmological solutions to $D=9$ can be easily generalised to arbitrary dimensions. The reverse of the 
procedure provides a mechanism for oxidising all the lower-dimensional solutions back to $D=10$ or $D=11$. Thus all the lower-dimensional solutions we obtained in section 2 can be viewed as ten or eleven dimensional solutions, and obtaining such lower-dimensional solutions provides a convenient algorithm for constructing and classifying sets of ten or eleven dimensional theories. Of course it is not guaranteed that the coordinate directions that are selected for this non-dynamical Kaluza-Klein compactification of the theory will actually shrink, rather than grow, as the cosmological solution evolves. Which of these occurs is a matter of calculation in the specific model in question. If it should turn out that some of the compactification directions actually expand with time, it becomes natural, from the ten-dimensional point of view, to include them in the spatial directions of the expanding universe. For example, we can construct a $p=2$ solitonic solution in four dimensions using a 2 -form field strength. From the four-dimensional point of view, we have a three-dimensional expanding universe, with one shrinking circle. However, if the 2-form field strength comes from the dimensional reduction of the 3 -form in $D=10$, oxidation of the four-dimensional solution to $D=10$ reveals that one of the six "compactifying" coordinates in fact expands, and hence gives rise to a four-dimensional expanding universe, which is no different from the $p=3, q=6$ solutions with $k=0$ in $D=10$ that we discussed earlier.

However, this does not imply that all the lower-dimensional solutions are nothing but reductions of already-known higher-dimensional solutions. As we saw earlier, the singlescalar $D=9$ solution with non-vanishing $\mu$ oxidises to a solution in $D=10$ that is not encompassed by the ansatz in section 2 . In fact as we saw in section 2 , a large number of solutions arise in lower dimensions that involve more than one field strength. The equations of motion have the form of a set of Liouville equations or $S L(N+1, R)$ Toda equations. The oxidation of these solutions provides a rich variety of cosmological solutions in $D=10$. It would be very interesting to analyse their cosmological significance.

\section{Conclusions}

In this paper we have made a rather extensive study of certain classes of cosmological solutions in $D=10$ string theory or M-theory. In particular, we began by constructing cosmological models in the $D$-dimensional toroidal compactifications of the string or M-theory, in which the metric takes the form (1). The relevant cosmological solutions correspond to cases where $p$-dimensional spatial sections that can be flat, spherical, or hyperboloidal expand in time, while an internal $q$-dimensional space undergoes a contraction. This space- 
time, with $D=p+q+1$ dimensions, can then be embedded in the original $D=10$ or $D=11$ theory by reversing the steps of the toroidal compactification to $D$ dimensions. This gives further dimensions that may be expanding or contracting, depending on the details of the solution. The models that would be of principal interest for cosmology are those where the total number of expanding spatial dimensions is 3. By dividing the process of constructing $D=10$ or $D=11$ solutions into these two stages, one can obtain rather broad classes of solutions with relative ease.

We examined some general features of the evolution of the metric scaling functions in some of the simpler solutions that we obtained. In certain cases, we found that the behaviour of the scale parameters was of the phenomenologically-desirable form, in which the "physical" spatial dimensions grow from a very small initial size to a large size at later times, while the additional "internal" dimensions shrink, or dynamically compactify. In particular, this kind of behaviour can arise in the $k=0$ and $k=-1$ models, where we find that the scale parameter $R$ of the physical spatial sections satisfies $R^{\prime \prime}>0$ at early times, and $R^{\prime \prime} \leq 0$ as $\tau \rightarrow \infty$, where the primes denote derivatives with respect to the comoving time. On the other hand, in the $k=1$ models the comoving time runs within a finite range, and the scale factor $R$ diverges at both ends of the interval. We also obtained large classes of more complicated solutions whose cosmological properties we did not examine in detail, including those corresponding to the $S L(N+1, R)$ Toda equations. It would be interesting to investigate the cosmology of these models further.

\section{Acknowledgement}

K.-W.X. is grateful to TAMU for hospitality in the early stages of this work.

\section{References}

[1] G.W. Gibbons and P.K. Townsend, Cosmological evolution of degenerate vacua, Nucl. Phys. B282 (1987) 610.

[2] R. Brandenberger and C. Vafa, Superstrings in the early universe, Nucl. Phys. B316 (1988) 319.

[3] I. Antoniadis, C. Bachas, J. Ellis and D. Nanopoulos, An expanding universe in string theory, Nucl. Phys. B328 (1989) 117. 
[4] B. Campbell, A. Linde and K. Olive, Does string theory lead to extended inflation?, Nucl. Phys. B355 (1991) 146.

[5] A. Tseytlin and C. Vafa, Elements of string cosmology, Nucl. Phys. B372 (1992) 443.

[6] M. Gasperini and G. Veneziano, Pre-big bang in string cosmology, Astropart. Phys. 1, (1993) 317.

[7] R. Brustein and G. Veneziano, The graceful exit problem in string cosmology, Phys. Lett. B329, (1994) 429.

[8] K. Behrndt and S. Forste, String Kaluza-Klein cosmology, Nucl. Phys. B430 (1994) 441.

[9] E.J. Copeland, A. Lahiri and D. Wands, Low energy effective string cosmology, Phys. Rev. D50 (1994) 4868.

[10] M. Gasperini, J. Maharana and G. Veneziano, Graceful exit in quantum string cosmology, Nucl. Phys. B427 (1996) 349.

[11] A. Lukas, B.A. Ovrut and D. Waldram, Cosmological solutions of type II string theory, hep-th/9608195.

[12] S. Mukherji, A note on Brans-Dicke cosmology with axion, hep-th/9609048.

[13] N. Kaloper, Stringy Toda cosmologies, hep-th/9609087.

[14] J.E. Lidsey, On the cosmology and symmetry of dilaton-axion gravity, gr-qc/9609063.

[15] G. Papadopolous and P.K. Townsend, Intersecting M-branes, Phys. Lett. B380 (1996) 273.

[16] A.A. Tseytlin, Harmonic superpositions of M-branes, Nucl. Phys. B475 (1996) 149.

[17] I.R. Klebanov and A.A. Tseytlin, Intersecting M-branes as four-dimensional black holes, B475 (1996) 179.

[18] J.P. Gauntlett, D.A. Kastor and J. Traschen, Overlapping branes in M-theory, hepth/9604179.

[19] N. Khviengia, Z. Khviengia, H. Lü and C.N. Pope, Intersecting M-branes and bound states, hep-th/9605077. 
[20] H. Lü, C.N. Pope and K.S. Stelle, Vertical versus diagonal dimensional reduction for p-branes, hep-th/9605082, to appear in Nucl. Phys. B.

[21] H. Lü, C.N. Pope, E. Sezgin and K.S. Stelle, Stainless super p-branes, Nucl. Phys. B456 (1995) 669.

[22] H. Lü and C.N. Pope, p-brane solitons in maximal supergravities, Nucl. Phys. B465 (1996) 127.

[23] H. Lü and C.N. Pope, $S L(N+1, R)$ Toda solitons in supergravities, hep-th/9607027, to appear in Int. J. Mod. Phys. A.

[24] H. Lü, C.N. Pope and K.-W. Xu, Liouville and Toda solitons in M-theory, hepth/9604058.

[25] G.W. Gibbons and R.E. Kallosh, Topology, entropy and Witten index of dilatonic black holes, Phys. Rev. D51 (1995) 2839.

[26] A. Anderson, An elegant solution of the n-body Toda problem, J. Math, Phys. 37 (1996) 1349. 